\title{
SOBRE LA PERTINENCIA DE LAS CATEGORÍAS CULTURALES EN LA ELABORACIÓN DE LAS REPRESENTACIONES DE LOS NUEVOS MOVIMIENTOS SOCIALES
}

\author{
Manuel Cárdenas Castro \\ Universidad Católica del Norte, Santiago, Chile \\ Roberto Rodríguez López \\ Universidad Complutense de Madrid, Madrid, Espanha
}

RESUMEN: Se presenta un estudio de las representaciones sociales sobre el movimiento antiglobalización (MAG) elaboradas por una muestra de obreros españoles. Para el análisis de dichas representaciones se ha utilizado un método de asociación libre de palabras a un conjunto de estímulos y un cuestionario de imagen del movimiento (que toma la forma de un diferencial semántico). Los resultados obtenidos a través de la asociación libre de palabras son analizados por medio de métodos multivariados (análisis de conglomerados, escalamiento multidimensional y análisis de correspondencias). Los resultados del cuestionario de imagen son resumidos en siete factores principales cuyas medias son comparadas según criterio de auto-categorización política. El principal efecto observado es el rechazo, por parte de los sujetos de la muestra, a utilizar las categorías culturales que en otros estudios aparecían como las más pertinentes para la elaboración de las representaciones sociales sobre el MAG.

PALABRAS-CLAVE: Representaciones sociales; movimiento antiglobalización; métodos multivariados.

\section{ABOUT THE PERTINENCE OF CULTURAL CATEGORIES \\ ON THE ELABORATION OF REPRESENTATIONS OF NEW SOCIAL MOVEMENTS}

ABSTRACT: This paper presents a study of the social representations on the anti-globalization movement (AGM) elaborated from a sample of Spanish workers. A method of free association of words as stimuli and an image of movement survey (that takes the form of a semantic differential) were used for the analysis of these representations. The results obtained by free association of words were analyzed by multivariated methods (hierarchical cluster analysis, multidimensional scaling and correspondences analysis). The results on the image survey were summarized in seven principal factors. The averages of these factors were compared according to the criterion of political self-categorization. The principal effect observed was a rejection, from a part of the subjects of the sample, in using the cultural categories that in other studies appeared as the most pertinent fo the elaboration of social representations about the anti-globalization movement (AMG).

KEYWORDS: Social representations; anti-globalization movement; multivariated methods.

Si partimos de la base de que en nuestra cultura existen ciertos temas que constituyen el fondo desde donde es posible estructurar el campo de los conocimientos ordinarios o cotidianos y desde los que es posible asignarles sentido, entonces tendremos necesariamente que reconocer que la misma construcción de una representación social (RS) sobre algún objeto está parcialmente condicionada por dicho fondo cultural. Estas serían concepciones profundamente enraizadas en lo social que funcionarían al modo de las imágenes arquetípicas y que empujarían a aprehender los objetos sociales en términos de esas determinadas concepciones. Estos "temas culturales" actuarían sobre la trayectoria de una representación funcionando como fuentes de ideas o como marcos colectivos desde donde toda RS sería elaborada (Moscovici \& Vignaux, 1994).

En estudios anteriores (Cárdenas \& Blanco, 2004, 2006) nos hemos venido encontrando de modo recurrente con ciertas categorías que los sujetos utilizan como dimensiones de juicio pertinentes a la hora de evaluar al movimiento antiglobalización. Un ejemplo de dichas cate- gorías es la dimensión política, entendida como una dicotomía entre izquierda y derecha. Ahora bien, no se trata ya sólo de la idea de que dicha dicotomía sea pertinente a la hora de realizar juicios sobre actores sociales en materias políticas, sino más radicalmente, de la idea misma de que la política sería un campo estructurado de forma dicotómica.

Es quizás por ello que corroboramos una y otra vez la pertinencia de la categorización política a la hora de evaluar a los movimientos sociales, como si el mero hecho de representar un objeto que se vislumbra en dicho campo invocara, de forma automática, los contenidos culturalmente arraigados y acumulados para dar forma a ese tipo de representaciones. De este modo, se piensa que sea la representación que sea, y tenga esta el contenido que se quiera, los criterios que se utilizan para darle forma o rellenarla serían establecidos de forma cultural. De lo anterior podría deducirse que su transformación sería sumamente lenta debido a que se encuentra arraigada en el fondo social, el que es dependiente a su vez de la propia estructura de la formación social a la que corresponde. 
$\mathrm{Si}$ asumimos que entre las principales funciones de la RS se encuentra la de simplificar para el uso cotidiano el complejo universo ideológico, entonces coincidiremos en que la RS trae a la mano un conjunto de "saberes" y de criterios mediante los cuales esta se elabora. Por otra parte, si asumimos que uno de los efectos de la RS es el de generar categorías (Abric, 1987; Pérez, Moscovici \& Chulvi, 2002), entonces coincidiremos en que aceptar o rechazar la llamada de un grupo que actúa en el campo político dependerá, entre otros factores, de la cercanía percibida con este y de los costes sociales que la identificación pueda acarrear, así como de los niveles de eficacia percibidos en el mismo. En este sentido, la elaboración de una RS implicaría la categorización del campo social y traería aparejada unas ciertas prescripciones conductuales para los miembros del grupo que la realiza en relación con las categorías derivadas del proceso representacional.

El estudio que aquí se presenta pretende acercarse a las RS que un grupo de obreros elabora del MAG, entendida como una forma de conocimiento socialmente elaborado y compartido, con una orientación práctica y enfocado a la construcción de una realidad común en un conjunto social (Jodelet, 1984). Además, se intentará indagar en los "temas culturales" que funcionan como marco desde donde se genera dicha representación, los cuales a su vez constriñen o restringen las posibilidades de acción social. Se trata de delimitar no sólo aquello que actualmente mediatiza las relaciones entre la muestra y el objeto de representación (la RS propiamente dicha), sino de un intento de acceder a los elementos culturales más permanentes que son salientes a la hora de elaborar una RS sobre los movimientos sociales en general. En este sentido, podemos considerar que la aceptación o rechazo del llamado a la acción que el MAG realiza dependerá de las RS (que a su vez sería elaborada desde los "temas culturales"), las que funcionarían como un "campo psicológico interno" (Galam \& Moscovici, 1991) que define los rangos dentro de los que es posible tomar una opción o decisión respecto de dicha interpelación.

\section{Método}

\section{Muestra}

La muestra quedó compuesta por un total de 53 sujetos, 22 hombres $(41,5 \%)$ y 31 mujeres $(58,5 \%)$, con una media de 37,08 años (desviación típica de 11,078), y siendo el rango de edades comprendido entre 19 y 59 años.

Los sujetos encuestados desempeñaban sus funciones como operarios en industrias del rubro textil y de alimentación, en la industria metalúrgica, como dependientes en tiendas de comercio minorista o como trabajadores no especializados en empresas de servicios. La mayor parte de ellos estaba en posesión de un contrato fijo, aunque casi todos los ligados al ámbito de servicios sólo tenía un contrato temporal.

Cabe consignar que para la mayor parte de los procedimientos que utilizaremos nuestro $\mathrm{N}$ corresponde a las palabras o asociaciones realizadas a los estímulos inductores. El criterio que hemos escogido para dejar de incorporar sujetos tiene que ver con una saturación de los términos asociados a los 11 estímulos o categorías propuestos (el número de sujetos será relevante sólo para las comparaciones entre grupos).

\section{Instrumento}

El instrumento utilizado consta de dos partes: una que hemos denominado cuestionario de imagen, y la segunda llamada asociación libre de palabras.

El cuestionario de imagen es una escala de 26 ítems que toma la forma de un diferencial semántico y que se puede resumir en siete factores: Evaluación, Actividad, Amplitud, Potencia, Estilo de Negociación, Estilo de Comportamiento y Convicción (Cárdenas \& Blanco, 2004). Además, hemos incorporado una pregunta sobre la autocategorización política de la muestra (izquierda, centro y derecha) que nos servirá para comparar las medias obtenidas tanto en el conjunto de la escala como en los factores y en los ítems de la misma.

Para la asociación libre de palabras hemos utilizado 11 términos como estímulo inductor. Los conceptos seleccionados fueron: Globalización, Antiglobalización, Orden, Protesta, Izquierda, Derecha, Mayoría, Minoría, Estudiante, Profesional y Obrero. A los sujetos se les pedía que asociaran libremente palabras a dichos términos. Este método tiene en su base ciertos supuestos generales: (a) la gente tiene un limitado número de significados sobre un objeto específico, lo que permite generar los diccionarios de significados; (b) los contenidos de la RS están estructurados jerárquicamente sobre la base de su similitud o distancia con otros significados; (c) la estructura de la RS está orientada por pocas dimensiones actitudinales, aquellas que se refieren a su núcleo (Doise, Clémence \& Lorenzi-Cioldi, 1993). En su conjunto, estos términos nos permitirán observar cómo se posiciona nuestra muestra respecto de los otros conceptos y grupos mencionados. La inclusión del estímulo Antiglobalización nos permitirá realizar un análisis sobre los significados asociados a este.

Sobre las palabras asociadas a los 11 estímulos se generó un diccionario sobre el que se realizó el análisis de conglomerados jerárquico y el escalamiento multidimensional. Para las palabras asociadas al concepto Antiglobalización se generó un diccionario especial sobre el que se realizó el análisis de correspondencias múltiple. Dichos diccionarios se han realizado siguiendo un procedimiento acorde con las clásicas reglas de análisis de contenido, esto es: 
(a) todas las palabras son reducidas a su forma masculino singular; (b) diferentes formas de la misma palabra son reducidas a la forma más común en el diccionario; (c) los adverbios y sustantivos son reducidos a su correspondiente adjetivo si este ya existe en el diccionario; (d) las combinaciones de palabras son reducidas al correspondiente adjetivo cuando esta existe en el índice. Las combinaciones a las que no correspondan adjetivos son mantenidas; (e) las jergas son reducidas al más cercano adjetivo posible; (f) algunas palabras que obviamente tienen el mismo sentido son reducidas a la más común palabra de la muestra, salvo cuando tenga varios sentidos posibles (Di Giacomo, 1980).

Estos Conceptos fueron seleccionados de modo tal que las polaridades existentes entre ellas se hicieran evidentes y los sujetos se vieran forzados a fijar una opción más cercana a cada uno de los polos de los pares.

\section{Resultados}

\section{Cuestionario de Imagen}

En la Tabla 1 pueden observarse las medias de respuesta y su correspondiente desviación típica para dicho cuestionario (la escala toma puntajes desde +3 a -3 y las puntuaciones positivas remiten en sentido del primer adjetivo de cada par).

Si nos centramos en los resultados obtenidos por medio del cuestionario de imagen, se observará que las características que esta muestra atribuye al movimiento antiglobalización manifiestan una tendencia general positiva, de aprobación, destacando los rasgos que lo describen como un movimiento muy activo, respetuoso, atrevido y abierto. Además, se le considera muy conocido y seguro de su acción, la que califican de objetiva, valiente, coherente y basada en principios morales. En definitiva, sería un movi- miento juzgado bastante positivamente. Quizás sea interesante señalar que el único puntaje medio negativo corresponde a la característica que se le atribuye de desconfianza, aunque encontramos otros bastante bajos que remiten a la idea de un movimiento poco simpático para los encuestados, sobre todo por que buena parte de la muestra lo considera levemente prejuicioso y minoritario. La Figura 1 nos muestra el perfil de puntuaciones para cada factor.

Como puede apreciarse los factores que alcanzan una media mayor son los referidos a Actividad $(1,36)$, Convicción $(0,937)$, Estilo de negociación $(0,885)$ y Potencia $(0,644)$. En su conjunto, estos factores nos remiten a una imagen del MAG que resalta los factores ligados a una práctica contundente (activo, atrevido, valiente, etc.), a un compromiso profundo con sus propias creencias (coherencia y seguridad) que se realiza de manera consistente, y a sus rasgos que resaltan su carácter negociador a la hora de dirigirse a la población (flexibilidad, respeto, generosidad y apertura). Otro factor que se resalta es el referido a la potencia y que implica una atribución de fortaleza para el MAG. Se puede apreciar que los puntajes medios para el conjunto de la muestra en todos los factores son positivos, aunque esto podría explicarse por la mayor presencia de sujetos que de autodenominan de izquierda y que tienden a sesgar los puntajes en sentido positivo, que es como venimos comprobando que estos sujetos tienden a evaluar al MAG.

Sabemos por estudios anteriores (Cárdenas \& Blanco, 2004, 2006) que el factor denominado adscripción política es el mejor discriminador del que disponemos a la hora de hacer aparecer las diferencias entre los sujetos de un mismo grupo al evaluar un movimiento social. Es por ello que hemos segmentado la muestra a fin de forzar la aparición de dichas diferencias. La Tabla 2 nos muestra las frecuencias para auto-categorización política.

Tabla 1. Estadísticos Descriptivos.

\begin{tabular}{|c|c|c|c|c|c|c|c|}
\hline & $\mathbf{N}$ & Media & Desv. Típ. & & $\mathbf{N}$ & Media & Desv. Típ. \\
\hline 01. Democrático-autoritario & 52 & 0,73 & 1,670 & 14. Agradable-desagradable & 51 & 0,29 & 1,847 \\
\hline 02. Tolerante-intolerante & 51 & 0,33 & 1,657 & 15. Objetivo-subjetivo & 50 & 0,80 & 1,750 \\
\hline 03. Coherente-incoherente & 50 & 0,80 & 1,852 & 16. Consistente-inconsistente & 50 & 0,64 & 1,699 \\
\hline 04. Seguro-inseguro & 50 & 1,06 & 1,621 & 17. Simpático-antipático & 52 & 0,23 & 1,676 \\
\hline 05. Flexible-rígido & 51 & 0,31 & 1,816 & 18. Activo-pasivo & 50 & 1,58 & 1,630 \\
\hline 06. Creíble-no creíble & 52 & 0,79 & 1,613 & 19. Conocido-desconocido & 53 & 1,40 & 1,446 \\
\hline 07. Progresista-conservador & 52 & 0,73 & 1,859 & 20. Bueno-malo & 50 & 0,88 & 1,769 \\
\hline 08. Minoritario-mayoritario & 53 & 0,06 & 1,994 & 21. Respetuoso-irrespetuoso & 51 & 1,47 & 1,901 \\
\hline 09. Confiado-desconfiado & 50 & $-0,28$ & 2,011 & 22. Eficaz-ineficaz & 50 & 0,44 & 1,656 \\
\hline 10. Atrevido-no atrevido & 52 & 1,40 & 1,729 & 23. Valiente-cobarde & 49 & 0,98 & 1,797 \\
\hline 11. Moral-inmoral & 50 & 0,82 & 1,781 & 24. Generoso-mezquino & 47 & 0,70 & 1,829 \\
\hline 12. Realista-no realista & 49 & 0,71 & 1,882 & 25. Abierto-cerrado & 51 & 1,14 & 1,950 \\
\hline 13. Desprejuiciado-prejuicioso & 49 & 0,12 & 1,523 & 26. Fuerte-débil & 51 & 0,69 & 1,655 \\
\hline
\end{tabular}




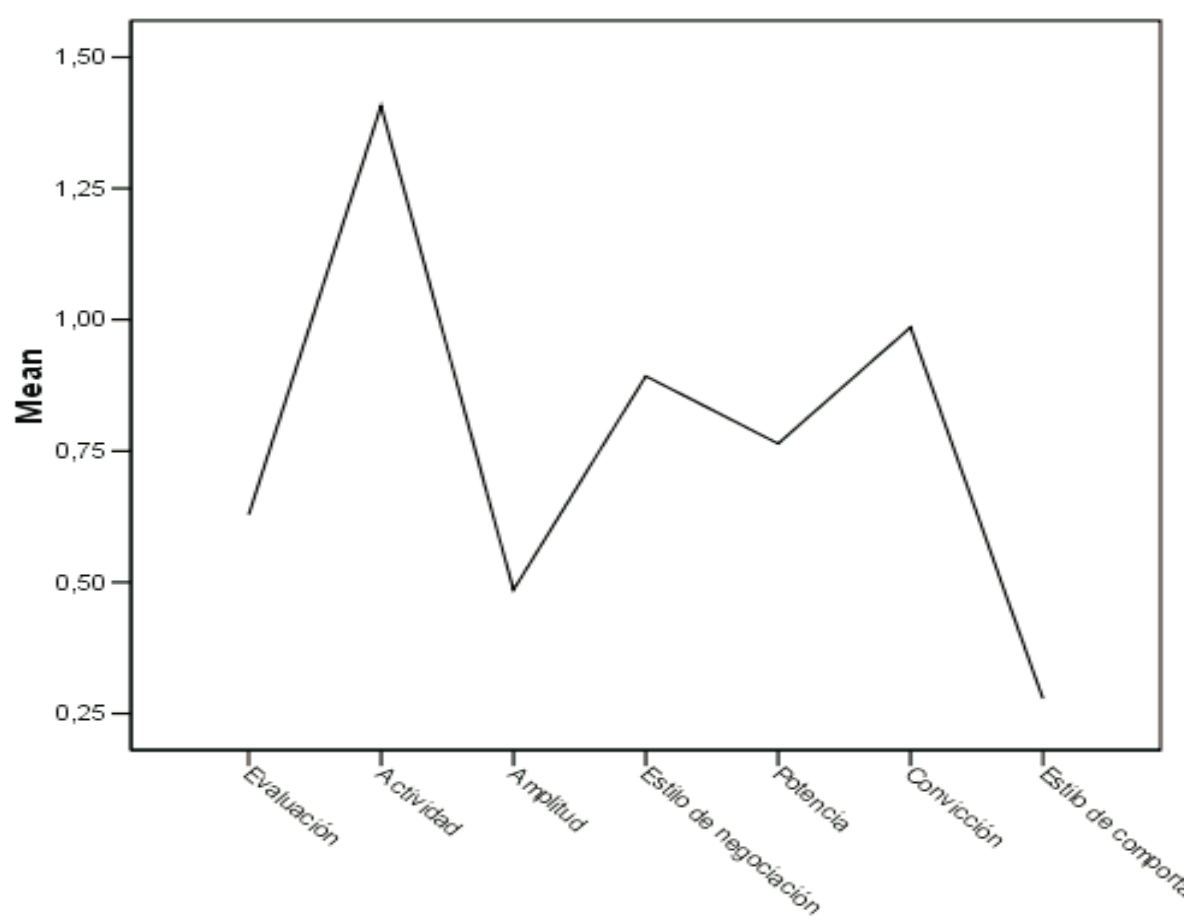

Figura 1. Perfil de puntuaciones para cada factor.

Tabla 2. Frecuencia para Auto-categorización Política.

\begin{tabular}{lcccc}
\hline & Frecuencia & Porcentaje & Porcentaje válido & $\begin{array}{c}\text { Porcentaje } \\
\text { acumulado }\end{array}$ \\
\hline Izquierda & 27 & 50,9 & 61,4 & 61,4 \\
Centro & 10 & 18,9 & 22,7 & 84,1 \\
Derecha & 7 & 13,2 & 15,9 & 100,0 \\
Total & 44 & 83,0 & 100,0 & \\
No responde & 9 & 17,0 & & \\
\hline Total & 53 & 100,0 & \\
\hline
\end{tabular}

Resulta interesante señalar que, como ya indicábamos con anterioridad, estos puntajes medios para el conjunto de la muestra tenderían a encubrir la importancia que la categoría "definición política" parece tener a la hora de explicar las adhesiones o rechazos frente al MAG. Contrariamente a lo esperado, podemos apreciar en la Tabla 3 que si bien los puntajes medios del grupo de centro son para varios factores visiblemente más bajos que los del grupo de izquierda y, sorprendentemente, de algunos del de derecha, estas diferencias no resultan significativas estadísticamente. En todo caso, nos parece llamativo el hecho de que el grupo de centro sea el que peor evalúa al MAG, así como las coincidencias en varios de los factores entre los trabajadores que se definen a sí mismos como de izquierda y derecha en varios de los factores (aunque con medias levemente inferiores para el segundo grupo).
Los datos de la muestra seccionada nos permiten afirmar que la imagen del MAG que elabora la muestra de izquierda remitiría principalmente a la idea de un movimiento bueno, agradable y por el que se siente simpatía, toda vez que se resalta su carácter flexible, abierto y respetuoso, su coherencia ideológica y su seguridad en el carácter moral que lo guía. Sería un grupo muy activo y conocido por las personas de la muestra, que resaltan entre sus rasgos fundamentales el valor y el arrojo de sus miembros.

En el grupo de centro encontramos que se ha ido construyendo una imagen del MAG que resalta ante todo su carácter activo, atrevido y valiente, y al que se considera muy conocido públicamente. Se le concede a sus argumentos una coherencia y un alto grado de convicción por parte de sus miembros. Eso sí, se le juzga como antipá- 
Cárdenas Castro, M.; Rodríguez López, R. "Sobre la pertinencia de las categorías culturales en la elaboración..."

Tabla 3. Estadísticos Descriptivos para los

7 Factores x Auto-categorización Política.

\begin{tabular}{|c|c|c|c|}
\hline Categoría & & Media & Desv. Típ. \\
\hline \multirow{7}{*}{ Izquierda } & Evaluación & 0,9792 & 1,67448 \\
\hline & Actividad & 1,6522 & 0,97334 \\
\hline & Amplitud & 0,6979 & 1,12041 \\
\hline & Estilo de negociación & 1,4062 & 1,43673 \\
\hline & Potencia & 0,9545 & 1,32206 \\
\hline & Convicción & 1,2778 & 1,47631 \\
\hline & Estilo de comportamiento & 0,3333 & 0,93735 \\
\hline \multirow{7}{*}{ Centro } & Evaluación & 0,0833 & 1,36931 \\
\hline & Actividad & 1,6563 & 1,12550 \\
\hline & Amplitud & 0,0750 & 1,06751 \\
\hline & Estilo de negociación & 0,3889 & 1,70069 \\
\hline & Potencia & 0,6750 & 1,17881 \\
\hline & Convicción & 0,9375 & 1,23744 \\
\hline & Estilo de comportamiento & 0,3056 & 0,82706 \\
\hline \multirow{7}{*}{ Derecha } & Evaluación & 0,1250 & 1,65643 \\
\hline & Actividad & 0,7500 & 1,29099 \\
\hline & Amplitud & 0,4583 & 0,60035 \\
\hline & Estilo de negociación & 0,3929 & 1,88114 \\
\hline & Potencia & 0,0000 & 1,43178 \\
\hline & Convicción & 0,1000 & 1,94936 \\
\hline & Estilo de comportamiento & 0,5417 & 1,30783 \\
\hline
\end{tabular}

tico y desagradable a la vez que ineficaz e inconsistente en su comportamiento. La tendencia a valorar al MAG menos positivamente por parte de este segmento de la población, podría deberse al marcado carácter autoritario que se percibe en él y a lo que se considera una actitud intolerante y prejuiciosa.

Finalmente, el grupo de derecha resalta (al igual que los otros dos) el carácter activo del MAG, así como su consistencia (a diferencia de los otros dos grupos que en este aspecto - estilo de comportamiento - puntúan más bajo). Para ellos el MAG es un grupo minoritario y poco potente, debido a su falta de realismo y objetividad. $\mathrm{Su}$ debilidad radicaría básicamente en que sería poco creíble.

Al comparar los puntajes medios para el conjunto de la escala (y utilizando como variable dependiente la autocategorización política) no encontramos diferencias significativas entre las sub-muestras $\left[\mathrm{F}_{(2,41)}=2,199 ; p=0.124\right]$, y estas tampoco aparecen para ninguno de los siete factores y tan sólo para 2 de los 26 ítems (18 y 19). En esta muestra, si bien es cierto es que estos datos deben ser analizados con sumo cuidado debido a la baja representatividad de los sujetos de la muestra $(N=53)$, las diferencias al nivel de la auto-categorización política no parecen tener efectos sobre la imagen que en su conjunto construyen sobre el MAG y que permitan explicar las adhesiones o los rechazos a dicho movimiento, ni la variabilidad al interior de la muestra.

Todo lo anterior nos indicaría que estos sujetos construyen la imagen de un movimiento novedoso en el campo social desde parámetros alejados de los referentes más tradicionales de la política y que podrían considerarse temas culturales (Moscovici \& Vignaux, 1994), los que nos remitirían a las creencias culturales de orden superior y desde las que es posible el acto de representar socialmente un objeto. La división izquierda-derecha sería uno de esos temas culturales desde donde toda representación social sobre un objeto "político" podría desarrollarse, y así lo habíamos corroborado en anteriores estudios con muestras diferentes (estudiantes y profesionales). De esta forma, resulta llamativo el hecho de que los obreros dese- 


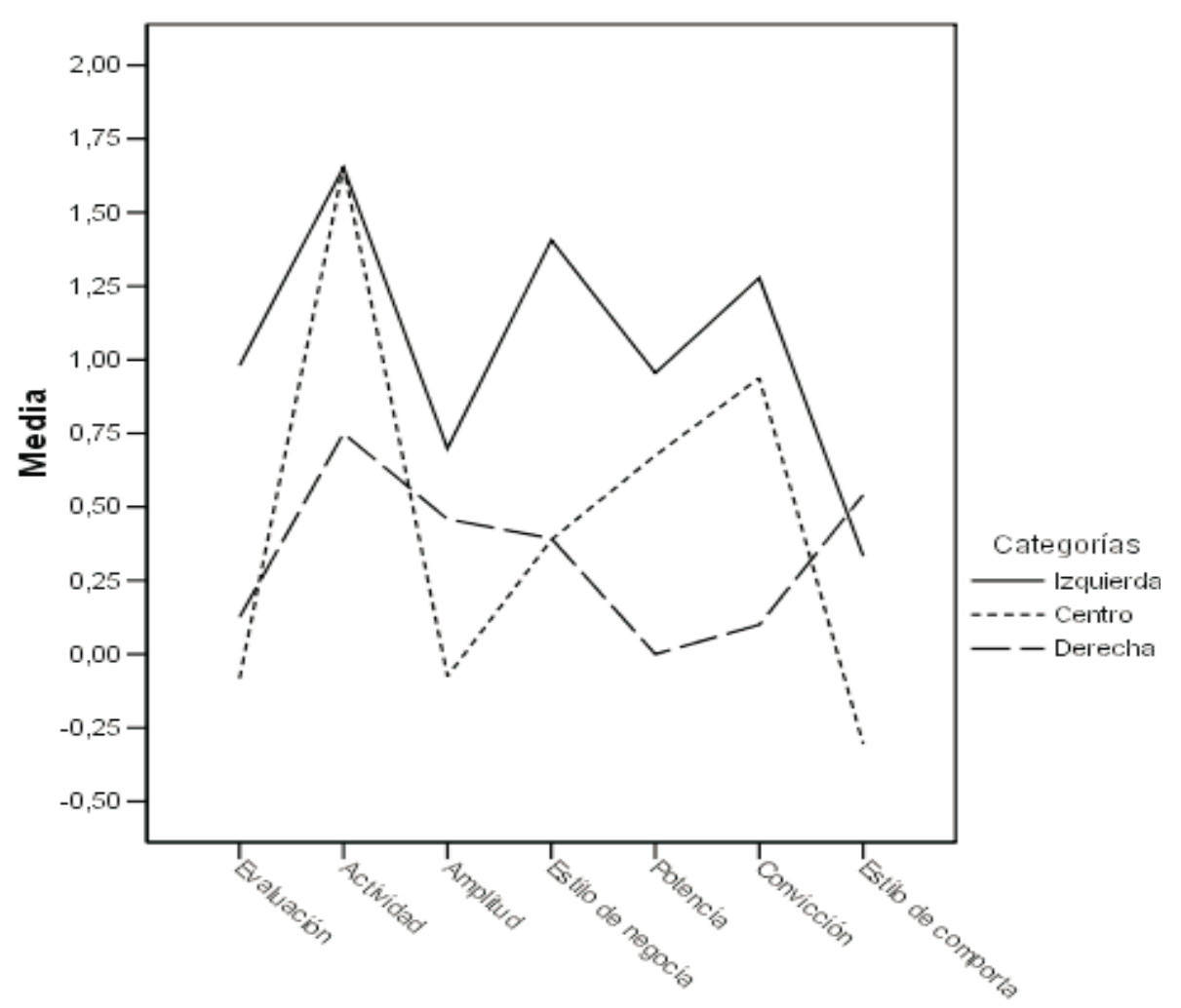

Figura 2. Perfil de puntuaciones para cada factor según auto-categorización política.

chen estos "temas culturales" y nos indiquen su intención de categorizar a partir de nuevas referencias o temas.

Volveremos sobre este tema más adelante, cuando analicemos los resultados del conjunto de los estudios sobre RS. Por ahora baste retener que la categoría política, que hasta ahora parecía ser un elemento esencial para comprender las posiciones adoptadas por las diferentes muestras, deja de ser funcional para nuestras interpretaciones y es negligida a la hora de realizar una representación sobre este objeto social. Las posiciones de la muestra no quedan explicadas por la variable que venimos comentando, y nos exige explicar esta variación. Este asunto lo retomaremos al final del artículo, una vez que terminemos de revisar los resultados de la segunda parte de nuestro instrumento.

\section{Asociación Libre}

La revisión de los resultados referidos a esta parte de nuestro instrumento nos muestra que para la asociación libre de términos a nuestros once estímulos la producción total de palabras para esta muestra de obreros ascendió a 1866 términos, lo que equivale a 35,21 conceptos por persona, y suponen un promedio de 3,2 términos por estímulo (para cada sujeto). De este total de palabras hemos reducido la cifra a 739 términos diferentes, mediante el procedimiento que ya detallamos, y que son los que se utilizarán como diccionario definitivo (este conjunto de palabras es el que realmente funciona como $\mathrm{N}$ en la planilla).
Para crear la matriz de distancias sobre la que hemos realizado nuestros análisis se ha utilizado una medida de similaridad para datos binarios (evalúan el grado de parecido o proximidad existente entre dos elementos expresados de forma dicotómica). En particular se ha utilizado el coeficiente Phi (de cuatro puntos). Esta medida de asociación es una versión binaria del coeficiente de correlación de Pearson (los valores de la matriz de distancia fluctúan entre -1 y 1). Esta matriz nos servirá para realizar los procedimientos de análisis de conglomerados jerárquicos $\mathrm{y}$ de escalamiento multidimensional.

El método de aglomeración que hemos utilizado es el de "vinculación intergrupos" que permite aprovechar la información de todos los miembros del conglomerado que se compara al calcular la distancia entre conglomerados como promedio entre todos los pares de elementos de cada conglomerado. La Figura 3 nos muestra el dendograma donde se observan las etapas del proceso de fusión y las distancias existentes entre los elementos fundidos en cada etapa.

Lo primero que llama la atención de este gráfico es la asimilación que se realiza del movimiento antiglobalización con la izquierda, a los que se suma también muy pronto la idea de protesta, situándolas dentro de un campo común. Tenemos constituido así un campo que evoca los mismos significados para estos términos, de modo que aparecen como fuertemente homologables. 


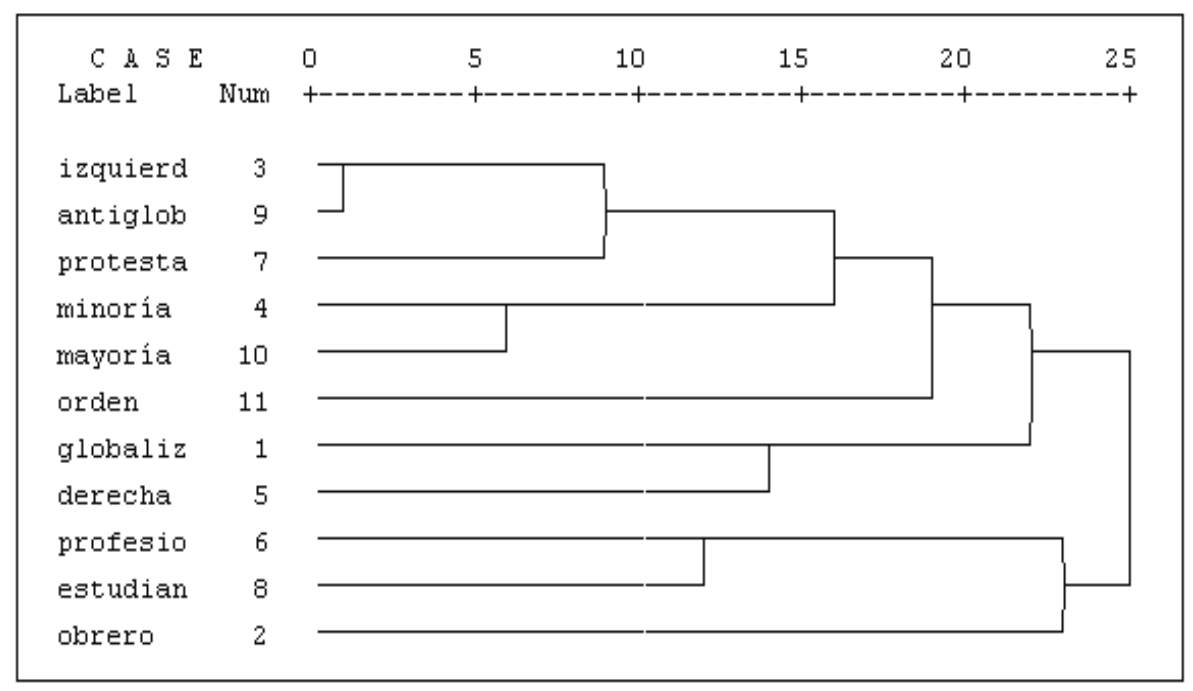

Figura 3. Dendograma.

Otro elemento interesante es el referido a la asimilación entre minoría y mayoría. Esta similitud percibida nos indica que algunos sujetos de la muestra se asumen formando parte de una minoría y otros como de una mayoría, y que en ambos casos utilizan valoraciones muy similares para el exogrupo que son las que hacen aparecer estos elementos como muy semejantes. Al parecer, este par de conceptos no nos es útil debido a su baja discriminación, ya que los sujetos no cuentan con una definición de la minoría como la que nosotros hemos venido utilizando en trabajos anteriores. Los sujetos no ligan la minoría solamente a la falta de poder social, sino mayormente al carácter numérico, que hace que la muestra con que trabajamos piense en sí misma integrada a una mayoría social, numéricamente superior. Por otra parte, es un elemento confuso, ya que por una parte se vierten calificativos muy duros contra lo que se considera una minoría económicamente poderosa, a la vez que se añaden los opuestos cuando los sujetos piensan en minorías raciales o étnicas. El gran problema es que en un sujeto suelen estar presentes al mismo tiempo ambos tipos de respuesta (pro o contra minoritaria o mayoritaria), ya sea que conteste para el caso de la mayoría o de la minoría. Lo mismo se puede decir que ocurre en el caso del cuestionario de imagen, donde el par minoría-mayoría deja de discriminar desde el momento en que a los sujetos no les proporcionamos una definición explícita de minoría. Por ello, no podemos considerar significativa la unión de la triada izquierda, antiglobalización y protesta, con la dupla mayoría-minoría. Si lo es en cambio la asimilación de los conceptos de derecha y globalización.

Podemos apreciar cómo se van formando sucesivamente dos polos diferenciados (que en otro sitio denominamos progresista y conservador) y al parecer gobernados por una lógica que consiste en dividir tajantemente el campo político en dos ámbitos separados, en el que solamente después se agregan a los colectivos sociales que analizamos más cerca de uno u otro polo. En todo caso, he aquí lo que esta configuración tiene de novedoso: los tres estímulos que refieren a los grupos con que venimos trabajando son percibidos como muy similares, aunque totalmente separados de todos los otros términos sobre los que se les consulta. En realidad, los obreros se ponen a sí mismos muy lejos de todo lo demás. Se puede observar que conglomeran muy tarde con los profesionalesestudiantes (que se agrupan bastante más temprano entre sî).

Si nos atenemos a los datos que nos entrega la muestra tendremos forzosamente que asumir que los obreros consideran que sus intereses y los elementos definitorios de su quehacer están totalmente ajenos a conceptos como globalización o antiglobalización, a izquierda o derecha, o a la protesta y al orden. Formarían una imagen de sí basada en atributos separados del campo políticamente trazado por ellos mismos y escogerían como compañeros de viaje a los estudiantes y a los profesionales (aunque reconociendo muy diferentes de los mismos).

Otro elemento que resulta llamativo es que la idea de orden se asocia más al polo progresista que al conservador. El mensaje podría ser el de que la noción de orden no depende de un determinado ideario político, sino que es independiente pero posible de ser asimilado a ambos (ambas concepciones sobre la política pueden ser ordenadas o propugnan una idea particular de orden), quitándole así la connotación de vigilancia y punición a que las otras muestras hacían referencia.

Retendremos para la discusión posterior la idea de que la división realizada en el campo deja de un lado el "mundo político" y del otro a los "grupos sociales". Una especie de escisión entre dos campos que desplaza a 
algunos de los supuestos destinatarios de la política a los confines de la misma. Eso sí, hemos de recordar que distinguen dentro del campo político, que no queda así indiferenciado, dos polos contrapuestos. Dentro del polo progresista se incorpora al MAG, la izquierda y la protesta. Muy cerca la idea de orden. Mayoría y minoría se unifican con este polo, pero como ya hemos mencionado no son buenos elementos a la hora de establecer diferencias. El otro polo vincula claramente a la derecha con la globalización.

A continuación, presentamos los datos obtenidos por medio del MDS y que nos permitirán indagar en las dimensiones subyacentes que permiten a los participantes de nuestra muestra realizar las diferenciaciones de que venimos hablando. Se trata de comprender ahora el porqué de estas dicotomización, las dimensiones mediante las que se realiza el procedimiento y situarlas en un espacio bidimensional.

En la Figura 4 se observan los resultados obtenidos por medio del procedimiento escalamiento multidimensional. En el se puede apreciar como el eje uno (horizontal) opone las palabras Obrero y Globalización, ubicadas en los extremos polares de dicho continuo. Muy cercana a Obrero se encuentran Estudiante y Profesional. En el campo semántico de Globalización se encuentra próximo el término Derecha. De este modo, la primera dimensión podría estar aludiendo a la oposición entre las categorías sociales y el proceso de globalización. Un proceso que sería comandado por, o que estaría ligado a, la derecha política y económica. De este modo, el primer eje opondría al proceso globalizador con los probables afectados por el mismo. Sería una dimensión que resalta el carácter alejado que cobra la globalización para los obreros, los que perciben también que es un proceso ajeno a los grupos profesionales y a los estudiantes.

El segundo eje opone los estímulos obrero y estudiante (palabra a las que se sumarían derecha e izquierda). Es decir, la dimensión de juicio con que se evalúa a este grupo social (estudiantes) nos habla de su mayor cercanía respecto de las tradicionales categorías utilizadas para dicotomizar el campo político. En este sentido los obreros podrían estar manifestándonos su deseo de ser evaluados al margen de estas adscripciones, o su creencia en que la categoría izquierda-derecha no sería pertinente para definirlos. Por otra parte, en esta dimensión sí que los obreros se encuentran cerca del concepto globalización y en oposición a estudiante, indicando con ello que la globalización es un proceso que los atañe directamente y que los afecta de modo mucho más evidente que al resto de los colectivos sociales.

Las dimensiones subyacentes utilizadas por los obreros para realizar sus juicios nos indican que, más allá de buscar un acomodo entre las categorías políticas, la pertinencia misma de dichas categorías sería puesta en cuestión. Se abandonaría el campo donde esa categorización es pertinente, se invocaría un terreno nuevo desde donde poder leer su ubicación. Ya no es más el cómo se ubican en la gradiente que va de izquierda a derecha, sino cómo la idea misma de izquierda o derecha no sería pertinente para los obreros.

Revisemos ahora el análisis de correspondencias múltiples sobre los términos más frecuentemente asociados al estímulo Movimiento Antiglobalización (para este análisis

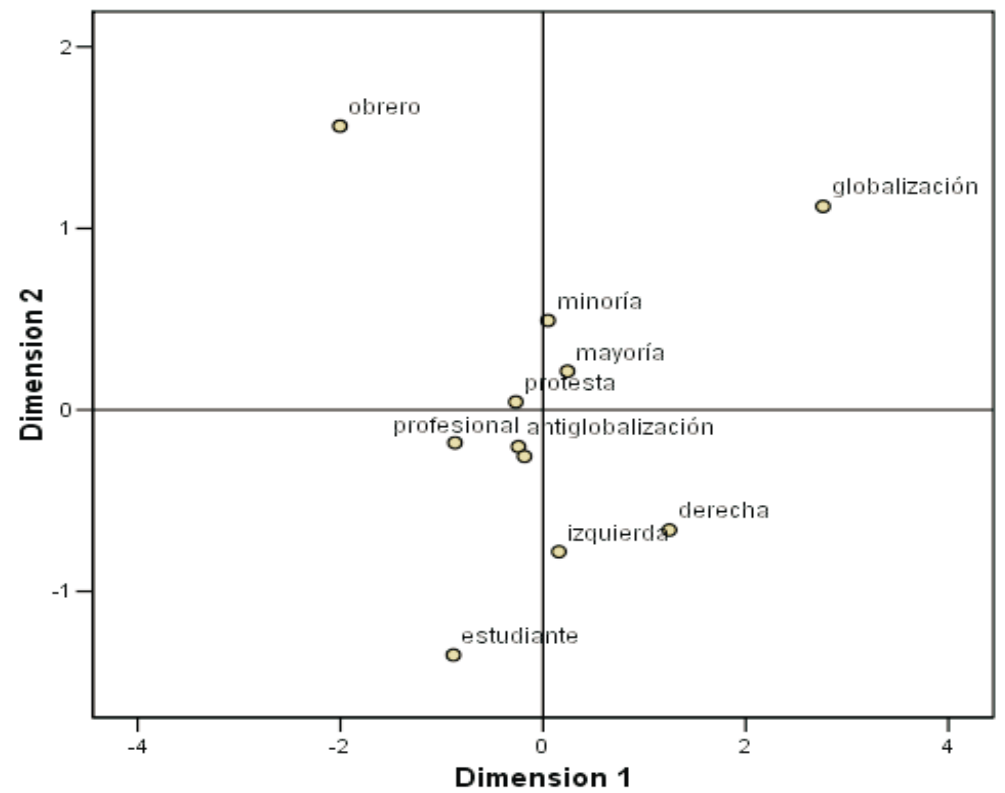

Figura 4. MDS. Representación del espacio para los once estímulos (obreros). 
hemos utilizado el programa estadístico STATISTICA 6.0).

La Tabla 4 nos muestra las frecuencias para los 14 términos asociados al estímulo "Movimiento Antiglobalización" que hemos utilizado como dimensiones activas para el análisis de correspondencias múltiples. Hemos preparado el material de igual forma que para los análisis anteriores. De este modo hemos agrupado el total inicial de palabras, que correspondía a 103 términos (con una media de 2,019 asociaciones por sujeto), reduciéndolo a 80. De entre estas se seleccionaron todas aquellas con una frecuencia de aparición superior a 3 , siendo por ello el número de asociaciones utilizadas de 14 (cuya suma de frecuencias de aparición es de 72).

Sobre este grupo de palabras asociadas al estímulo Antiglobalización se realizó el análisis de correspondencias, utilizándolas como elementos activos para crear las dimensiones o factores subyacentes. Se han seleccionado las tres primeras dimensiones, cuyos valores propios asociados explicarían en conjunto un $42,68 \%$ de la inercia total $(16,57 \%, 13,94 \%$ y $12,17 \%$ respectivamente).

Como puede observarse, la dimensión uno opone las palabras Vandalismo y Anticapitalismo, siendo la estructura de representación más cercana al primer término la formada por las palabras Desorden y Manifestación. Para el segundo polo las palabras más allegadas son Defensor, Izquierda y Justicia (Figura 5). Consideramos que esta dimensión pone en juego, a la vez, los elementos descrip-

Tabla 4. Frecuencias para Estímulo Antiglobalización (Obreros).

\begin{tabular}{cc}
\hline Palabra & Frecuencia \\
\hline Protesta & 10 \\
Oposición & 8 \\
Movimiento & 8 \\
Idealismo & 7 \\
Avance & 6 \\
Justicia & 5 \\
Defensor & 5 \\
Necesario & 5 \\
Anticapitalista & 3 \\
Desorden & 3 \\
Coherente & 3 \\
Izquierda & 3 \\
Manifestación & 3 \\
Vandalismo & 3 \\
\hline Total & 72 \\
\hline
\end{tabular}

tivos del movimiento y los mecanismos por los que pone en marcha su acción (se trata del estilo de comportamiento o de sus estrategias de acción). En su conjunto, la dimensión sería de identidad, pero aludiría tanto a la definición como a las formas prácticas de expresión. Se trataría de un movimiento social de izquierda que lucharía por la defensa de los derechos que el capitalismo niega. Esta lucha se traduciría en la búsqueda de mayor justicia y contra los efectos perversos generados por el capitalismo.

La segunda dimensión opone las palabras Anticapitalismo y Defensor. El campo representacional de la primera palabra del par anterior lo conforman los términos Avance y Justicia. Para crear el campo semántico de Anticapitalismo se utiliza la palabra Izquierda (y un poco más alejadamente Oposición). Estaríamos nuevamente ante un eje de identidad, pero que también remitiría a los elementos de totalidad, es decir, al horizonte que apunta la acción colectiva del MAG.

La tercera dimensión (Figura 6) por la que la muestra elabora su imagen del MAG sería de carácter evaluativo, en tanto se juzga al MAG como un movimiento idealista. También se remite a su condición política de izquierda, que claramente tendrá consecuencias en la decisión de sumarse o no a su acción colectiva (recordemos que nuestra muestra se instala, o al menos eso pretende, en un terreno que considera más allá de la dimensión izquierda-derecha). El otro polo de dicha dimensión nos indica del carácter coherente que se le asigna y, aunque un poco alejado de este término, la palabra Necesario viene a servir de corolario en esta caracterización.

En términos generales, podemos apreciar que las dimensiones utilizadas para juzgar al MAG son de carácter evaluativo y descriptivo, apuntando en el sentido de definir lo que es (por medio de sus prácticas y atributos más estereotípicos), aunque intentando captar el sentido de su lucha y el horizonte al que apunta su acción. Eso sí, el MAG es definido más por lo que se cree que es que por aquello a lo que se opone. En este sentido podemos suponer que se ha realizado un intento de captar los elementos centrales de su discurso y una valoración de su acción. Un discurso coherente que apunta a lograr una transformación social tendente a la obtención de mayores cotas de justicia, por la defensa de los derechos de las personas y que implique un genuino avance social. Se trataría de un movimiento de izquierda que lucha contra el capitalismo de una manera activa aunque nada ordenada y que estaría integrado por sujetos que en ocasiones recurren al vandalismo para como medio de lucha para alcanzar sus objetivos.

\section{Discusión}

Si nos centramos en los resultados obtenidos, podemos observar la aparición de ciertos patrones con los que ya 


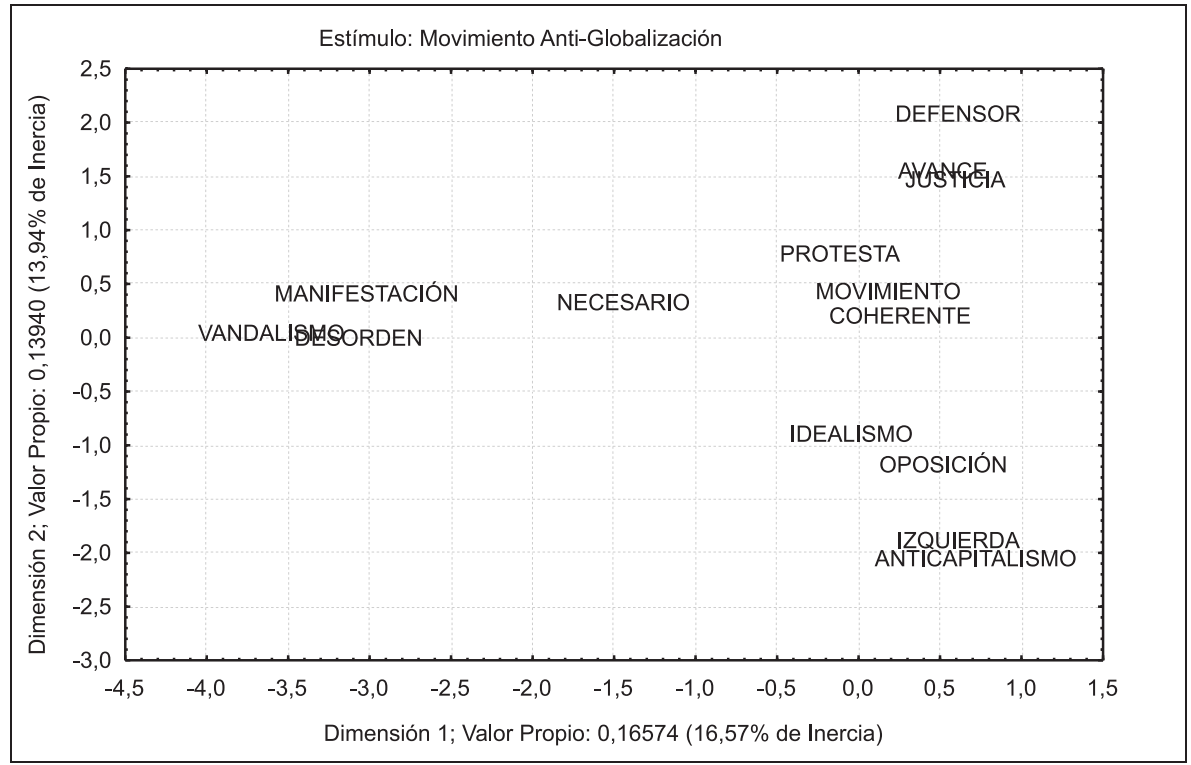

Figura 5. Análisis de Correspondencias. Dimensiones 1 y 2.

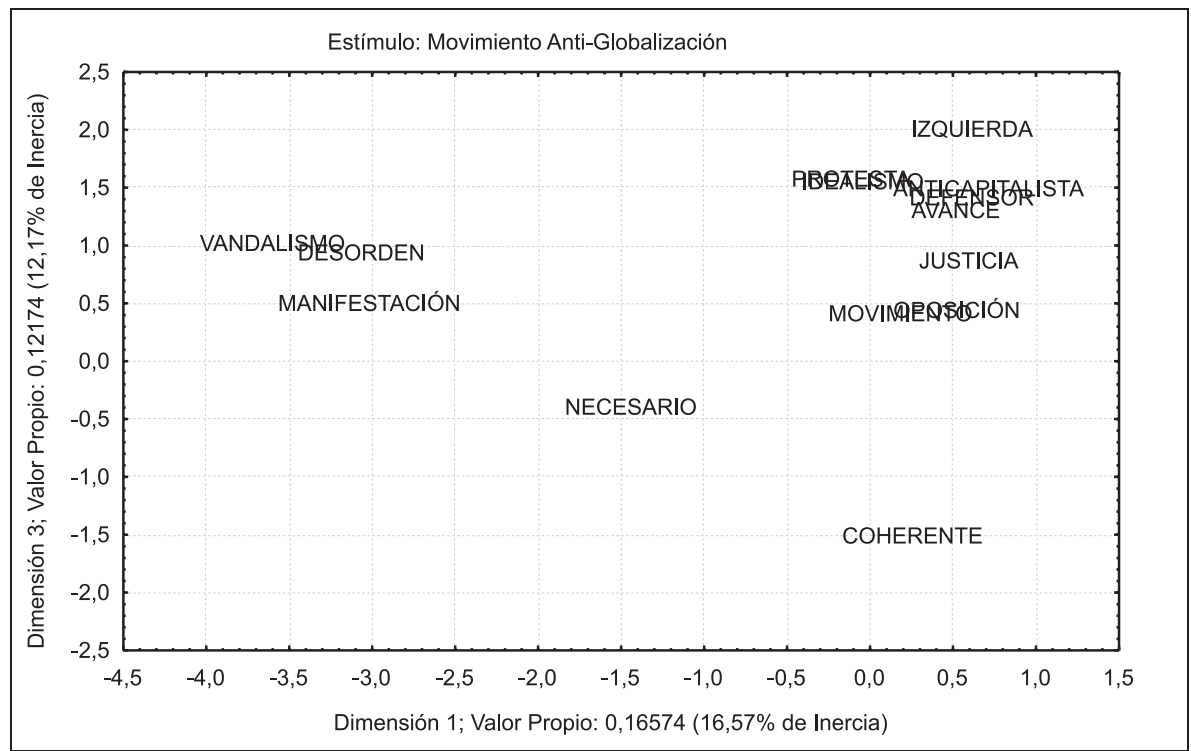

Figura 6. Análisis de Correspondencias. Dimensiones 1 y 3.

nos habíamos familiarizado por estudios anteriores. Se trata de una concordancia, entre diferentes muestras, en las dimensiones utilizadas para elaborar las RS sobre el MAG. Lo anterior podría explicarse por el hecho de que bastaría la sola incitación a representar un movimiento social (que por definición actúa en el ámbito de lo político o de lo público) para que se sitúe a este, de forma inmediata, en un campo político dicotomizado entre izquierda y derecha. De ahí la pertinencia esperada de la variable auto-categorización política, ya que si yo mismo me ubico dentro de uno de los campos señalados elaboraré una imagen del MAG como endogrupo o exogrupo, lo que evidentemente afecta la valoración que haga del mismo.

Es aquí donde nos encontramos con el elemento que a nuestro parecer es el más destacado en la muestra con la que trabajamos: a pesar de aparecer dicha dicotomía, los sujetos realizan un intento por ubicarse fuera de ella. Ya no de un lado o de otro, a favor o en contra, sino fuera del terreno delimitado por ella. Es decir, el reconocimiento de la categoría como dimensión de análisis existe, pero no es menos real la negativa de utilizarla, de aparecer fuera del área que dicho terreno especifica, aún cuando ya se ha aceptado auto-etiquetarse políticamente. Es decir, los obreros no sólo rechazan el uso de una categoría social asentada para aplicarla sobre sí (ciertamente segmenta el campo y reconoce la categoría en su seno, pero se ubica a sí mismo al margen de ella), sino que además se niega a aplicarla en la valoración del movimiento social analizado (recordemos que no encontramos diferencias significativas entre las sub-muestras). Es decir, se rechaza como per- 
tinente la categoría política para definirse a sí o al MAG, pero se le reconoce como un "tema cultural" a la mano a la hora de categorizar.

En términos muy generales los obreros de nuestra muestra evalúan positivamente al MAG, atribuyéndole con mucha fuerza una serie de características positivas. Se le considera un movimiento muy seguro de sí y muy atrevido, toda vez que conocido y bueno. Se destaca su carácter respetuoso y abierto, su actitud decidida y valiente, orientada por profundas convicciones morales. En este sentido no hay muchas diferencias con las otras muestras sobre el conjunto de la escala y para la totalidad de los sujetos de la muestra. El único elemento evaluado de forma negativa sería la actitud del MAG (calificada como desconfiada).

Cuando segmentamos la muestra de obreros nos enteramos de que la imagen de los sujetos de izquierda viene a ser mucho más positiva y que por ello ha servido para encubrir las diferencias internas de la muestra al sesgar las medias en sentido de su valoración (diferencias que no son significativas y que señalan que los tres grupos tienen, de forma más o menos marcada, una imagen general del MAG que es positiva). Para los sujetos de izquierda, el MAG destaca en tres factores (Actividad, Estilo de negociación y Convicción) que suelen indicar que se les representa como un movimiento muy atrevido y valiente, consistente en su acción y de marcado reconocimiento público. Su modo de relacionarse con otros grupos sería percibido como flexible, abierto y respetuoso. Sus ideas serían juzgadas como coherentes y mostrarían un alto grado de seguridad en ellas.

Llama la atención el hecho, bastante inesperado, de que el grupo que se define como de centro manifieste una tendencia a evaluar más negativamente al MAG que el mismo grupo de derecha. Ellos concuerdan en señalar al MAG como un grupo muy activo, atrevido y valiente, pero lo consideran más bien autoritario e intolerante, toda vez que desconfiado, inconsistente e ineficaz. Para el grupo de derecha en cambio estos atributos negativos no están presentes y coinciden, aunque en un tono mucho más moderado, con los atributos destacados por los sujetos de izquierda.

La explicación a esta extraña coincidencia parece encontrarse en el hecho de que los sujetos de derecha juzgan como poco potente al MAG, es decir, como un movimiento débil y claramente alejado de la realidad. Si en otro sitio afirmábamos que la potencia parecía ser un elemento relevante como predictor de las adhesiones a un movimiento social, entonces en el caso de que la potencia se considere menguada se puede simpatizar con un movimiento que es ajeno al propio campo en la medida en que se le atribuye una escasa capacidad de alterar el rumbo de los acontecimientos. De este modo, se debería reformular la hipótesis antes señalada y decir que: en caso de encontrarse en el propio campo del movimiento social, la potencia percibida será el elemento que permita predecir las conductas de adhesión o los intentos de diferenciación respecto del mismo. Y al revés, en caso de que el movimiento sea percibido como formando parte del exogrupo, se podrá elaborar una imagen de él más favorable sólo en la medida en que la potencia percibida sea baja.

Los análisis realizados sobre la segunda parte del instrumento nos indican que se asimila al MAG con la izquierda (aunque recordemos que lo que antes se realizaba de forma conciente aquí toma la forma de una asociación libre y por ello menos conciente) y que estos dos términos se unen de modo muy rápido con el concepto de protesta. Observamos que también se asocia a la derecha con la globalización. El problema surge a la hora de ponerse a sí mismos y a los otros grupos (profesionales y estudiantes) en relación con estos polos semánticos. Es el momento en que surge el rechazo a la identificación, a asumir aquellas categorías rescatadas del fondo cultural y que hemos denominado "temas". Se reconoce la existencia de la dicotomía izquierda-derecha, la exigencia cultural de interpretar desde estos parámetros, pero se rechaza (ya sea por desconocimiento de cómo ubicar a los actores implicados o por negativa a asumir esos parámetros como definitorios de su identidad) su utilización. Decíamos que conscientemente y de modo individual los sujetos de la muestra aceptan auto-categorizarse políticamente (para ser más exactos 44 de los 53 sujetos contestan a nuestra pregunta por la auto-categorización), pero que a la hora de situar a su grupo dentro del campo definido por estos parámetros de un modo menos meditado se topan con problemas.

Creemos, a modo de hipótesis, que podría tratarse de un intento de construir su identidad grupal desde un ámbito diferente, aunque no necesariamente opuesto, del definido por este tema tan arraigado culturalmente. Afirmamos que la noción misma de obrero puede haberse vuelto problemática debido a los inmensos cambios en la dinámica del trabajo en los tiempos de la globalización. De aquí podría deducirse que la misma categoría de obrero comienza a redefinirse y a encontrar sus señas de identidad desde un ámbito diferente del definido por la adscripción política, pero que debería estar ligado a las transformaciones a las que ya hemos hecho referencia.

Al intentar indagar en las dimensiones utilizadas para elaborar las diferenciaciones precedentes nos encontramos con que la primera de ellas alude a la contraposición entre los tres grupos que nos han servido de muestras y el concepto de globalización. Es decir, la globalización (íntimamente ligada a la derecha) es un proceso que se da a espaldas de los grupos sociales, es un proceso comandado desde un ámbito totalmente alejado de los grupos analizados y que resaltaría la idea de que se toman decisiones que afectan a estos grupos sin participación de los mismos en ellas. La segunda dimensión es, como en las 
otras muestras, eminentemente política, pero a diferencia de los otros grupos aquí es utilizada de modo diferente. Los obreros estarían alejados tanto de la izquierda como de la derecha (y no deja de ser llamativo en sujetos que han consentido en ubicarse a sí mismos en una de estas categorías).

Finalmente, al intentar profundizar en las relaciones internas existentes entre los términos asociados al MAG nos encontramos que al menos tres dimensiones son pertinentes para representarlo. Estas dimensiones aluden a aspectos evaluativos y descriptivos. Es decir, se intenta captar al MAG como objeto social definiendo lo que es y aquello a lo que aspira, así como los medios que utiliza para alcanzar dichos objetivos. De este modo, la primera dimensión referiría a los elementos descriptivos tanto del movimiento mismo como de sus formas de expresión. En este sentido sería un movimiento de izquierda que lucharía contra la injusticia y por la defensa de los derechos conculcados por el capitalismo. Ahora bien, sería propio de su acción la manifestación casi siempre desordenada, y en algunos casos, difícil de distinguir del mero vandalismo. La segunda dimensión intentaría captar los elementos de identidad del MAG por la vía de precisar sus contenidos, la totalidad u horizonte al que apunta. Así, su deseo de justicia y su defensa de los derechos se considerarían un avance respecto de otros movimientos. Sería un movimiento necesario para poner límites a un capitalismo desbordado. Finalmente, la tercera dimensión sería de carácter netamente evaluativo. El MAG sería juzgado como un movimiento de protesta, ligado a la izquierda y de carácter idealista. Su discurso y su acción son juzgados como coherentes y necesarios.

Ahora bien, si consideramos que los elementos del núcleo de una representación son cuantitativamente y cualitativamente diferenciables del resto de los significados, entonces podemos suponer que un indicador de la centralidad de los elementos del núcleo sería la saliencia de dichos significados. Así, las palabras más frecuentemente nombradas serían aquellas de carácter central (en sentido cuantitativo), así como aquellas que mayor conectividad tengan (sentido cualitativo). Si bien el procedimiento que utilizamos no nos permite observar la conectividad entre los elementos ni el tipo de vínculos existente entre estos, podemos considerar las dimensiones subyacentes como elementos de centralidad con que los sujetos elaboran un RS. Para el caso de nuestra muestra el MAG sería representado como un movimiento social que se opone por medio de la protesta al capitalismo, con una actitud idealista y que significaría un avance respecto de los movimientos sociales precedentes. Estos serían los elementos definitorios del MAG. Se definiría por "oposición a”, por el conflicto que busca instalar en el campo social. Los elementos periféricos de esta RS aludirían a su carácter violento o vandálico, a su vinculación a la izquierda tradi- cional, a su coherencia interna, a su afán de justicia, etc. Como podemos apreciar, el vino nuevo se ha puesto en odres viejos. El carácter de novedad del MAG es subsumido en los caracteres más generales de todo movimiento social. Una vez más vemos como ya objetivado lo abstracto en una imagen concreta, se ancla lo nuevo en lo ya conocido.

\section{Referências}

Abric, J-C. (1987). Cooperation, competition et représentations sociales. Cousset, Suisse: Del Val.

Cárdenas, M., \& Blanco, A. (2004). Las representaciones sociales del movimiento antiglobalización. Revista de Psicología Política, 28, 27-54.

Cárdenas, M., \& Blanco, A. (2006). Representación e influencia de los nuevos movimientos sociales (el movimiento Antiglobalización). Psicología Social, 21(2), 153-169.

Di Giacomo, J-P. (1980). Intergroup alliances and rejections within a protest movement (Analysis of the social representations). European Journal of Social Psychology, 10, 329-344.

Doise, W., Clémence,A., \& Lorenzi-Cioldi, F. (1993). The quantitative analysis of social representations. London: Harvester Wheatsheaf.

Galam, S., \& Moscovici, S. (1991). Towards a theory of collective phenomena: Consensus and attiutude changes in groups. European Journal of Social Psychology, 21, 49-74.

Moscovici, S., \& Vignaux, G. (1994). Le concept de Thêmata. In C. Guimelli (Ed.), Structures et transformations des représentations socials (pp. 25-72). Paris: Delachaux et Niestlé.

Jodelet, D. (1984). The representation of the body and its transformations. In R. Farr \& S. Moscovici (Eds.), Social representations (pp. 211-238). London: Cambridge University Press.

Pérez, J-A., Moscovici, S., \& Chulvi, B (2002). Natura y cultura como principio de clasificación social. Anclaje de representaciones sociales sobre minorías étnicas. Revista de Psicología Social, 17(1), 51-67.

Manuel Cárdenas é Psicólogo, Doutor em Psicologia Social pela Universidad Autónoma de Madrid. Atualmente é professor da Escuela de Psicología de la Universidad Católica del Norte Endereço para correspondência: Escuela de Psicología, Universidad Católica del Norte, Av. Angamos 0610, Antofagasta, Chile. jocarde@ucn.cl

Roberto Rodríguez é Psicólogo, Doutor em Psicologia Social pela Universidad Complutense de Madrid.

\section{Sobre la pertinencia de las categorías culturales en la elaboración de las Representaciones de los Nuevos Movimientos Sociales}

Manuel Cárdenas Castro \& Roberto Rodríguez López

Recebido: 26/02/2007

$1^{a}$ Revisão: $30 / 08 / 2007$

Aceite Final: 24/09/2007 